\title{
Novel approach of rigid bronchoscopy concurrent with cesarean section and bronchial arterial embolism for patients with massive hemoptysis during pregnancy: case reports and literature review
}

\author{
Xue Yang ${ }^{1}$, Wei Ma ${ }^{2}$ Xin $\mathrm{Shi}^{3}$, Xiao Sun ${ }^{4}$, Yumei Wei ${ }^{4}$, Ziguang Yan $^{5}$, Shuangling $\mathrm{Li}^{6}$ \\ ${ }^{1}$ Department of Geriatrics, Peking University First Hospital, Beijing, China; ${ }^{2}$ Department of Cardiology, Peking University First Hospital, Beijing, \\ China; ${ }^{3}$ Department of Anesthesiology, Peking University First Hospital, Beijing, China; ${ }^{4}$ Department of Obstetrics and Gynecology, Peking \\ University First Hospital, Beijing, China; ${ }^{5}$ Department of Interventional Radiology and Vascular Surgery, Peking University First Hospital, Beijing, \\ China; ${ }^{6}$ Department of Critical Care Medicine, Peking University First Hospital, Beijing, China \\ Correspondence to: Shuangling Li, MD. Department of Critical Care Medicine, Peking University First Hospital, Beijing 100034, China. \\ Email: lishuangling888@hotmail.com.
}

\begin{abstract}
Massive hemoptysis can be life-threatening and is frequently encountered in clinical practice, but rare during pregnancy. There have been limited case reports of massive hemoptysis in pregnancy in patients with conditions such as Takayasu's arteritis, bronchiectasis, bronchial carcinoid tumor, and tuberculosis. The most important management is early control of the hemorrhage and airway protection. We report on 2 patients at 33 and 27 gestational weeks who were admitted to the emergency department because of massive hemoptysis. Therapeutic rigid bronchoscopy with the application of high-frequency jet ventilation was performed under general anesthesia during cesarean section to control potential bleeding and stabilize the airway; this was then followed by bronchial artery embolization (BAE) postsurgically. The lives of both mothers and infants were saved. At the 16- and 11-month follow-ups, the patients showed no symptoms. To our knowledge, this is the first report on the application of therapeutic rigid bronchoscopy concurrent with cesarean section in order to protect the airway and reduce the side effects of the subsequent treatment for both mother and fetus in hemoptysis cases. By reporting these cases and conducting a literature review, we present a novel treatment method for massive hemoptysis in pregnant patients that may improve patients' outcomes.
\end{abstract}

Keywords: Massive hemoptysis; pregnancy; rigid bronchoscopy; bronchial artery embolization (BAE); case reports

Submitted Mar 14, 2020. Accepted for publication Jan 29, 2021.

doi: 10.21037/atm-20-2502

View this article at: http://dx.doi.org/10.21037/atm-20-2502

\section{Introduction}

Massive hemoptysis is a life-threatening emergency. There is currently no consensus on a uniform volume threshold for hemoptysis to be considered massive, with suggestions ranging from $100 \mathrm{~mL} / 24$ hours to more than $1,000 \mathrm{~mL}$ per 24 hours $(1,2)$. The unreliable estimation of volume and differences in cardiopulmonary function reserve have led to differing definitions of massive hemoptysis that depend on the amount of expectorated blood, which refers to hemoptysis that causes airway obstruction and asphyxia $(1,3)$. There have been limited case reports of massive hemoptysis in pregnant patients with conditions such as Takayasu's arteritis (4), bronchiectasis (5), bronchial carcinoid tumor (6), and tuberculosis (7). The disruption of the high-pressure bronchial circulation or the explosion of pulmonary circulation, leading to the pathologically high pressures of the bronchial vasculature, accounts for most of the adverse circumstances arising during pregnancy from changes in intravascular volume (8). During pregnancy, massive hemoptysis may impair gas exchange, causing 
hypoxia in the mother and the fetus, and may cause maternal hemodynamic instability due to blood loss. The ability to identify the etiology of hemoptysis and the effectiveness of controlling the hemorrhage and airway with multidisciplinary efforts are key to successfully managing this life-threatening condition.

In this article, we present 2 cases of massive hemoptysis that occurred during pregnancy, which were treated with multidisciplinary therapy. The combination of simultaneous therapeutic rigid bronchoscopy and cesarean section under general anesthesia, followed by bronchial artery embolization (BAE) after surgery is reported here for the first time. We also provide a literature review and suggest a new management solution suitable for patients with cryptogenic massive hemoptysis that can be undertaken with a planned cesarean section. We present the following case in accordance with the CARE reporting checklist (available at http://dx.doi.org/10.21037/atm-20-2502) (9).

\section{Case presentation}

\section{Case 1}

A 29-year-old woman was admitted to the maternity department of our hospital with massive hemoptysis at 33 weeks' gestation on September 21, 2018. The amount of blood loss was estimated at approximately $100 \mathrm{~mL}$ within 1 hour before admission. She denied coughing, night sweats, or fever and had been previously well. She was a preschool teacher, did not smoke cigarettes, and had no known tuberculosis contact. On physical examination at admission, she was anxious, and except for tachycardia, her blood pressure (BP) was $110 / 60 \mathrm{mmHg}$ with a pulse rate of 114 beats per minute (BPM). Her admission laboratory data are presented in Table 1. In addition, there was no serological evidence of respiratory infection. An echocardiogram was normal. An electron-nasopharyngolaryngoscope test confirmed no active bleeding of the upper airway.

On the second day of admission, she had another episode of hemoptysis and developed breathlessness. On examination, she was alert, her BP dropped to $96 / 56 \mathrm{mmHg}$, and her pulse was $78 \mathrm{BPM}$. Physical examination revealed a relatively diminished breath-sound in the right chest. Arterial blood gas (ABG, with supplemental oxygen via a nasal catheter of $5 \mathrm{~L} / \mathrm{min}$ ) analysis showed a $\mathrm{pH}$ of $7.47, \mathrm{PCO}_{2} 29 \mathrm{mmHg}$, $\mathrm{PO}_{2} 60 \mathrm{mmHg}, \mathrm{HCO}_{3}{ }^{-} 23.2 \mathrm{mmol} / \mathrm{L}$, lactate $1.0 \mathrm{mmol} / \mathrm{L}$, and $\mathrm{SaO}_{2} 92 \%$. In addition, her hemoglobin dropped from 100 to $82 \mathrm{~g} / \mathrm{L}$. The mediastinal window of the chest computed tomography (CT) showed a thrombus obstructing the bronchus intermedius, the right middle lobe bronchus, and the right lower lobe bronchus (Figure 1). Considering the hemodynamic instability of the patient, she was transferred from the maternity ward to the intensive care unit (ICU), but hemoptysis persisted. We initiated the administration of high-flow nasal cannula oxygen therapy with $65 \%$ oxygen at a flow rate of $40 \mathrm{~L} / \mathrm{min}$ at a temperature of $34{ }^{\circ} \mathrm{C}$. Other medications included tranexamic acid (2.5 g within 24 hours) and dexamethasone to promote fetal lung maturation. A multidisciplinary team (MDT) including personnel from the ICU, interventional surgery, anesthesiology, respiratory intervention, and maternalfetal medicine departments met with the patient's family. Therapeutic rigid bronchoscopy and cesarean section under intravenous general anesthesia were performed simultaneously. A healthy baby girl weighing 2,390 g with APGAR (appearance, pulse, grimace, activity, and respiration) scores of 2 and 7 at 1 and 5 minutes, respectively, was delivered. An 8.5 Storz rigid bronchoscope was placed with high-frequency jet ventilation (HFJV) during the cesarean which revealed evidence of blood clots in both the anterior and posterior segments of the right upper lobe bronchus as well as the bronchus intermedius (Figure $2 A, B, C$ ). These were extracted by a cryoprobe and occluded with gauze. After grasping the gauze, a bronchial tamponade was performed with a gelatin sponge, and after no ongoing persistent bleeding was ensured, a double-lumen endobronchial tube (DLT) was intubated to protect the airway from potential bleeding (Figure 2). The patient then underwent emergency bronchial arteriography with a $5 \mathrm{~F}$ Cobra catheter (Terumo, Tokyo, Japan). Selective angiograms of the bronchial artery demonstrated multiple areas of contrast staining in the right lower lobe and a distal connection featuring a bronchialpulmonary arterial fistula (Figure $3 A$ ). The right lower lobe bronchial arterial branches were then embolized with 560 $710 \mu \mathrm{m}$ of gelatin sponge particles, and a postembolization right bronchial arterial angiography confirmed satisfactory occlusion of the treated vessels (Figure 3B). After the procedure, the patient was immediately returned to the ICU. Given her stable situation, the DLT was removed and replaced with a single-lumen endotracheal tube on the first postpartum day. Rigid bronchoscopy was repeated on the fourth day, and both large blood clots and the gelatin sponge were removed from the bronchi with cryoprobes. However, slow but persistent bleeding was noted from the distal to the proximal portions of the B6-10 lung segments while the clots were removed. Local hemostatic therapy using iced 
Table 1 Laboratory data on admission

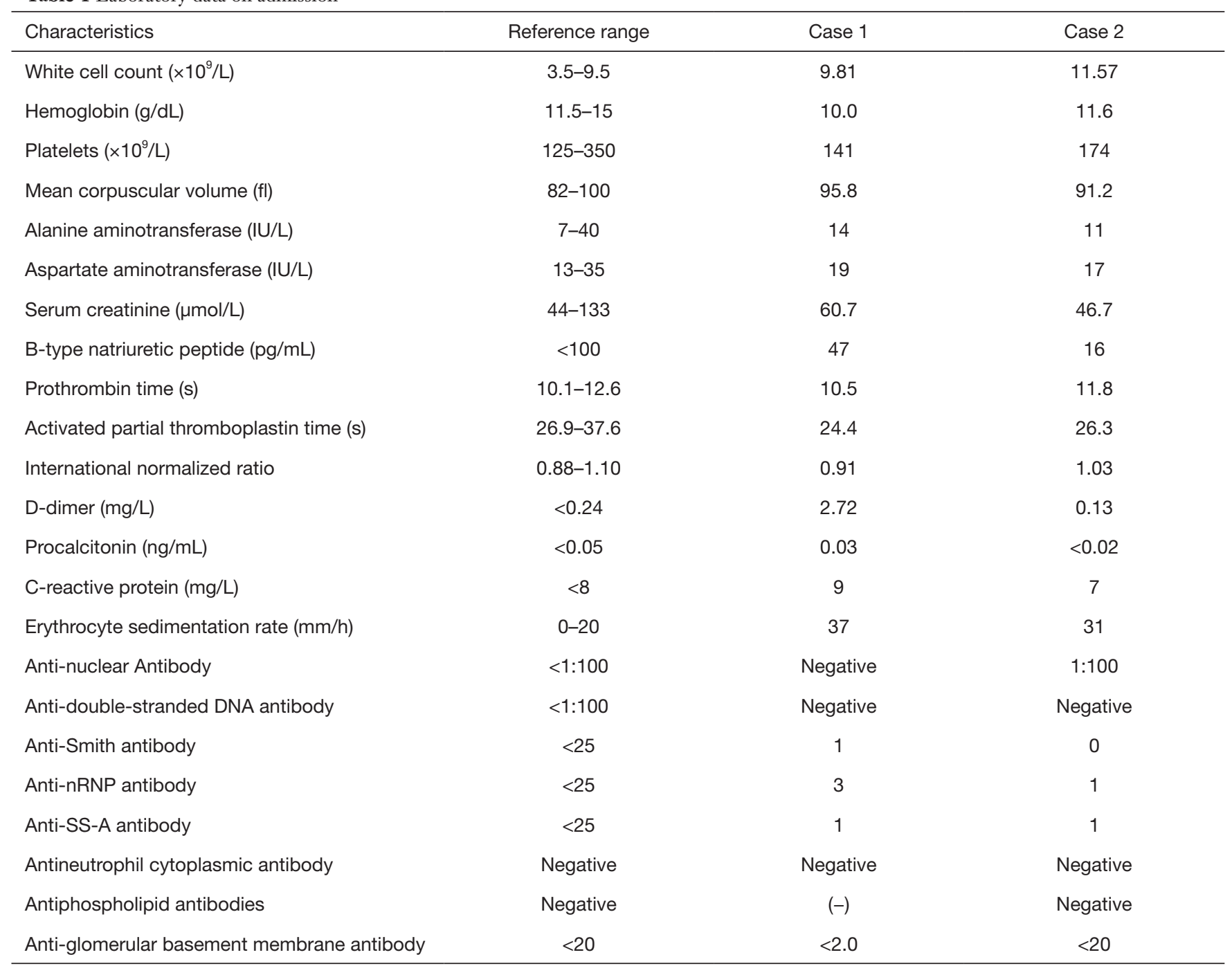

saline and thrombin (500 IU) failed, so we then performed subsequent gelatin sponge filling.

Based on suggestions from the multidisciplinary consultation, the patient accepted repeated therapeutic bronchoscopy with interventional surgery on stand-by. Cryotherapy was repeated successfully on the sixth day to extract the gelatin sponge and blood clots of the right lower lobe (Figure 2D,E,F). The patient was on low doses of propofol, dexmedetomidine, and remifentanil hydrochloride for sedation and analgesia while undergoing intubation. Management to prevent further atelectasis included chest physiotherapy and administration of ambroxol. The tracheal tube was pulled out on the seventh postpartum day, and the patient was then transferred to the maternity ward. She was observed for the subsequent 8 days with no further episodes of hemoptysis and was then discharged. The patient was doing well at 16 months after the treatment, and the infant's development was normal.

\section{Case 2}

A 37-year-old woman at 27 weeks' gestation with a history of untreated pulmonary hypertension $(\mathrm{PH})$ had experienced several weeks of hemoptysis and dyspnea in the third trimester of pregnancy when admitted on March 10, 2019. An initial episode had occurred 8 years earlier when she was pregnant at 16 gestational weeks, and at that time, she underwent an abortion. A subsequent right heart 

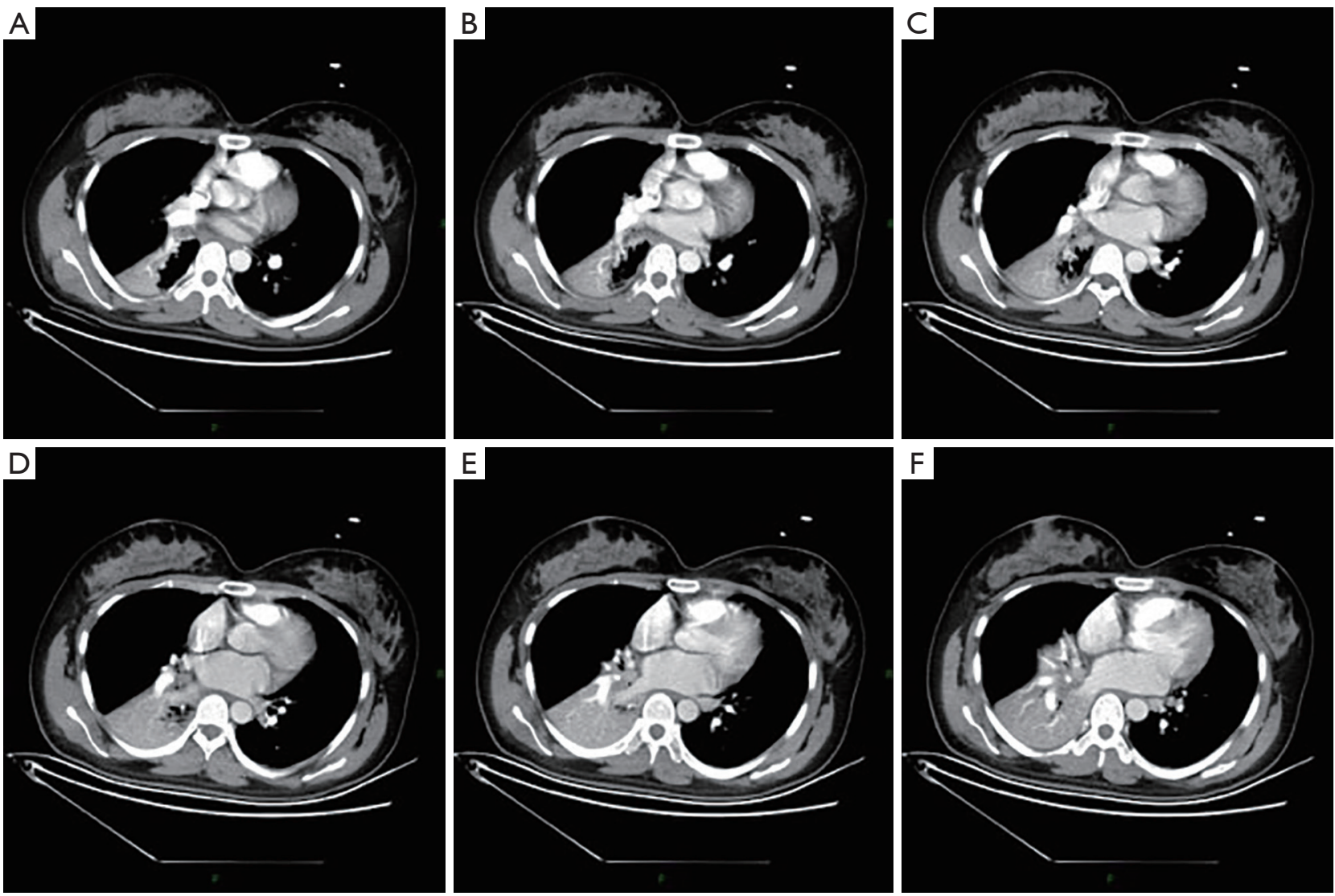

Figure 1 On mediastinal window images, chest computed tomography demonstrates a thrombus obstructing the bronchus intermedius, along with the right middle lobe bronchus and the right lower lobe bronchus being associated atelectasis of the right middle lobe and the right lower lobe

catheterization (RHC) was performed at the outpatient clinic of Peking Union Medical College Hospital that demonstrated elevated pulmonary artery pressure (PAP); however, the report was lost and we did not know the exact PAP measurement. Her sister died at 32 years from cyanosis and hemoptysis. The patient denied receiving any medications and reported no hemoptysis occurrence since her initial episode. One month earlier, she had an untreated episode of scant hemoptysis, and 1 day before the admission, she had coughed up more than an estimated $100 \mathrm{~mL}$ of blood over several hours. A physical examination at the time of the patient's presentation revealed a gravid female in no acute distress. Her vital signs were stable. Her laboratory tests are presented in Table 1. An echocardiogram showed a severely dilated right ventricle (RV) with an elevated $\mathrm{RV}$ pressure $(75.3 \mathrm{mmHg})$ resulting in a D-shaped left ventricle (LV; Figure 4A). The LV ejection fraction was $77.3 \%$. A transthoracic echocardiogram with an agitated saline "bubble study" demonstrated evidence of a right-to-left shunt suggesting pulmonary arteriovenous malformation (PAVM) (Figure 4B).

Intravenous tranexamic acid was initiated, and the amount of hemoptysis decreased. However, on the sixth day, the patient once again coughed up an estimated $100 \mathrm{ml}$ of blood. A cesarean section concurrent with rigid bronchoscopy with HFJV under intravenous general anesthesia was then performed, and a male baby with APGAR scores of 6 and 8 at 1 and 5 minutes, respectively, was delivered. The rigid bronchoscopy revealed blood clots in the opening of the bilateral lower lobe bronchus and in the right middle lobe bronchus which were removed with cryoprobes (Figure 5). The extracted blood clots revealed a bronchial cast (Figure 6). Ice saline and thrombin (500 IU) were used for local hemostasis after the clots were removed. An endotracheal tube was inserted after assuring no active bleeding. Further bronchial arteriography demonstrated enlargement and 


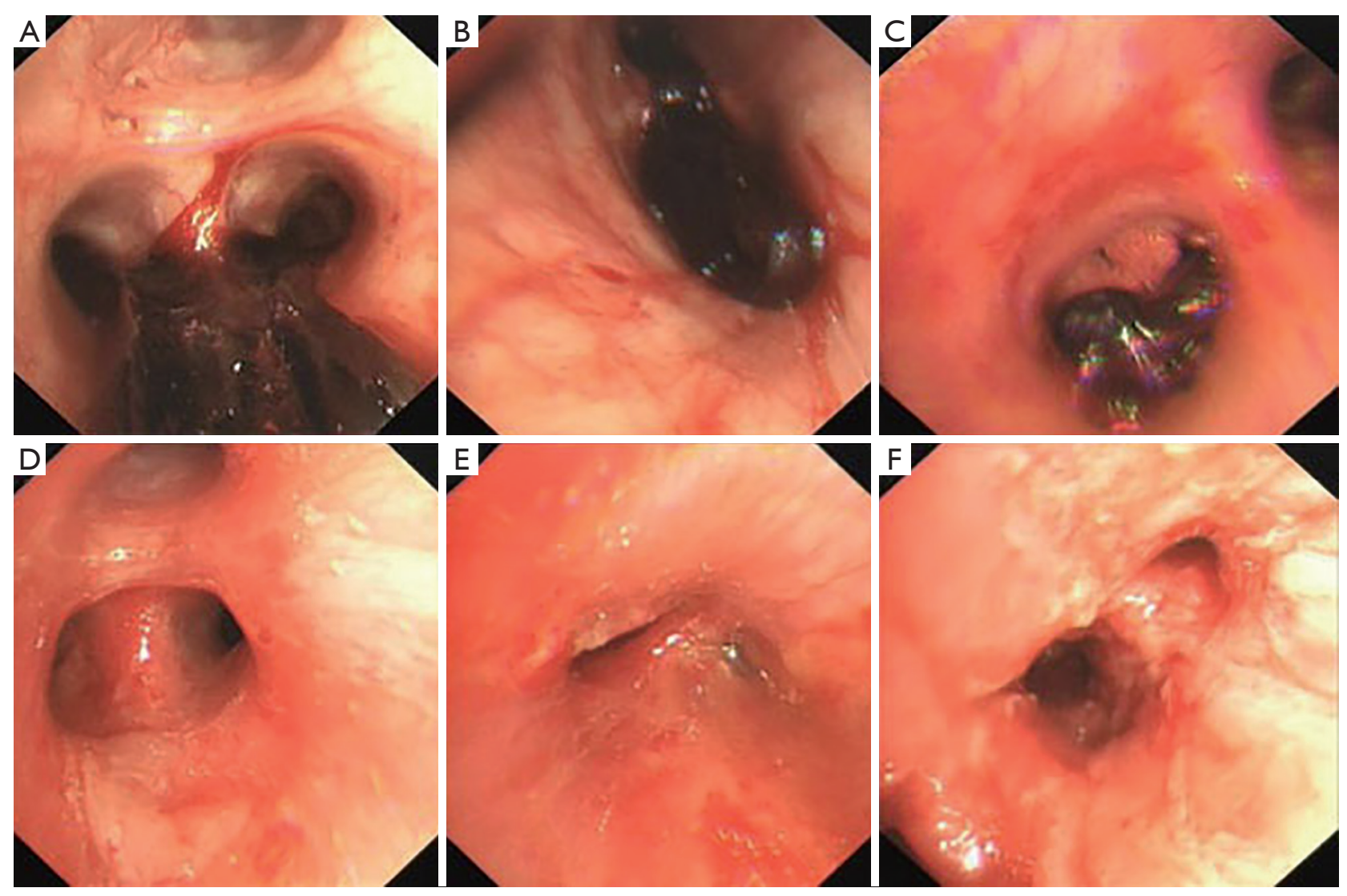

Figure 2 Rigid bronchoscopy images before and after surgery. Blood clots in the right upper lobe bronchus (A) and the bronchus intermedius (B) were each frozen and packed with a gelatin sponge (C). View of the bronchus after cryotherapy and thrombectomy to remove the gelatin sponge and blood clots (D,E,F).
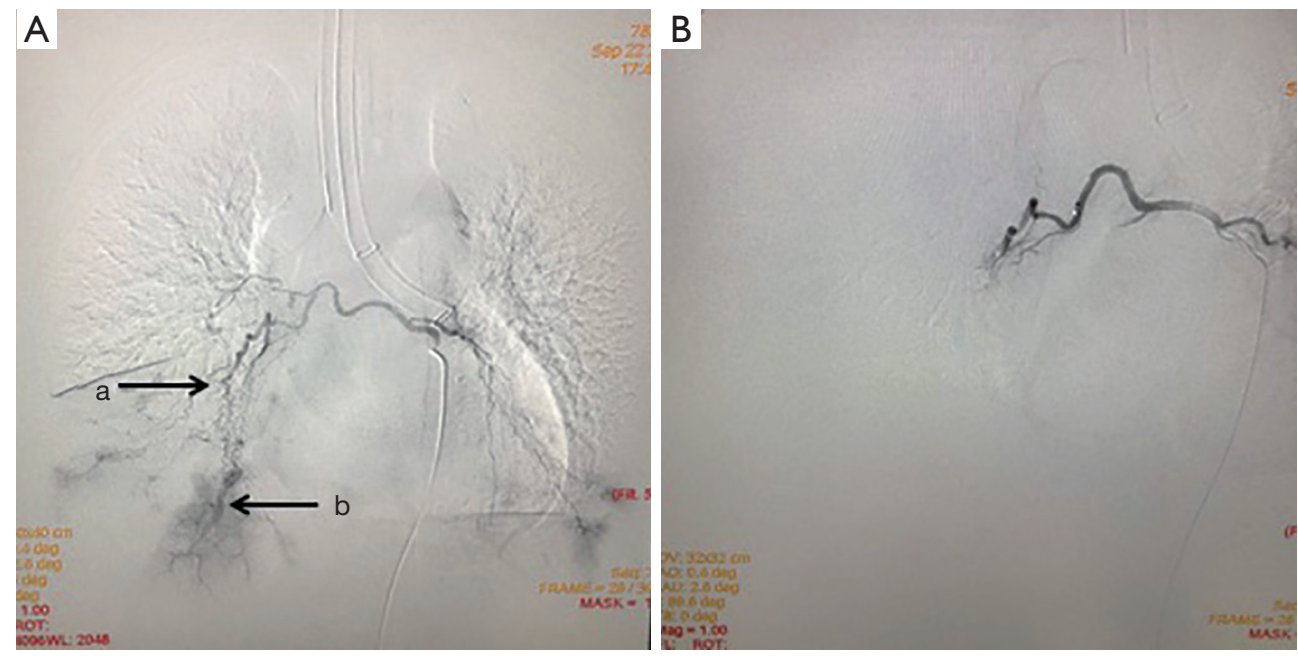

Figure 3 Selective angiograms of the bronchial artery before and after embolization. (A) Pre-embolization: Selective angiograms of the bronchial artery demonstrate a descending branch (a) supplying the bronchial tree, and faint opacification of a bronchial-to-pulmonary artery shunt featuring a bronchia-pulmonary arterial fistula (b). (B) Post-embolization: Selective catheterization of the bilateral bronchial arteries shows complete occlusion of the connection with no flow to the pulmonary artery. 

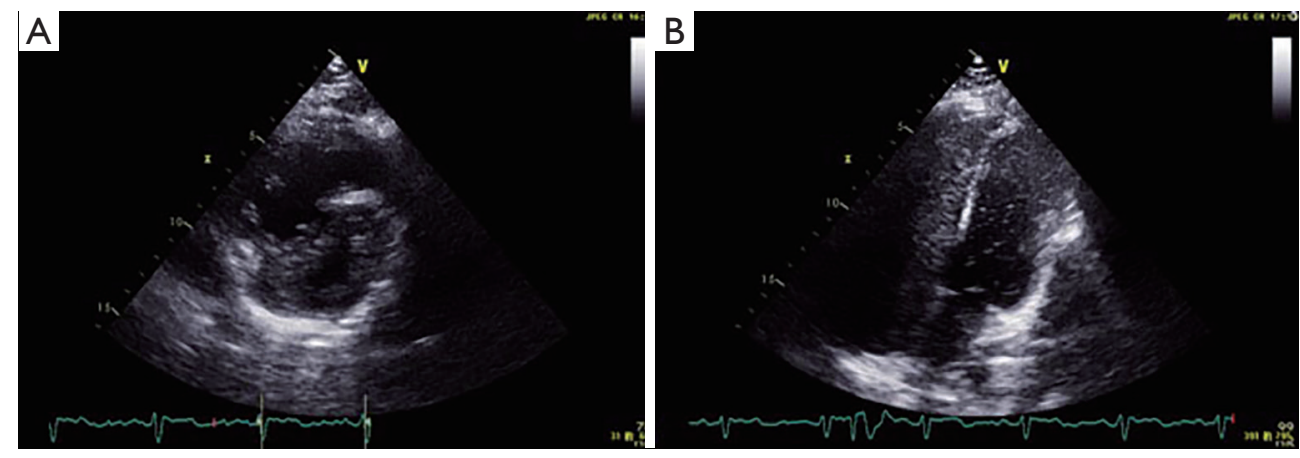

Figure 4 Echocardiography of the patient. (A) Echocardiography shows a compressed left ventricle forming a D-shape. (B) Agitated saline contrast transthoracic echocardiography demonstrates bubbles appearing in the left ventricle after their presence in the right atrium.

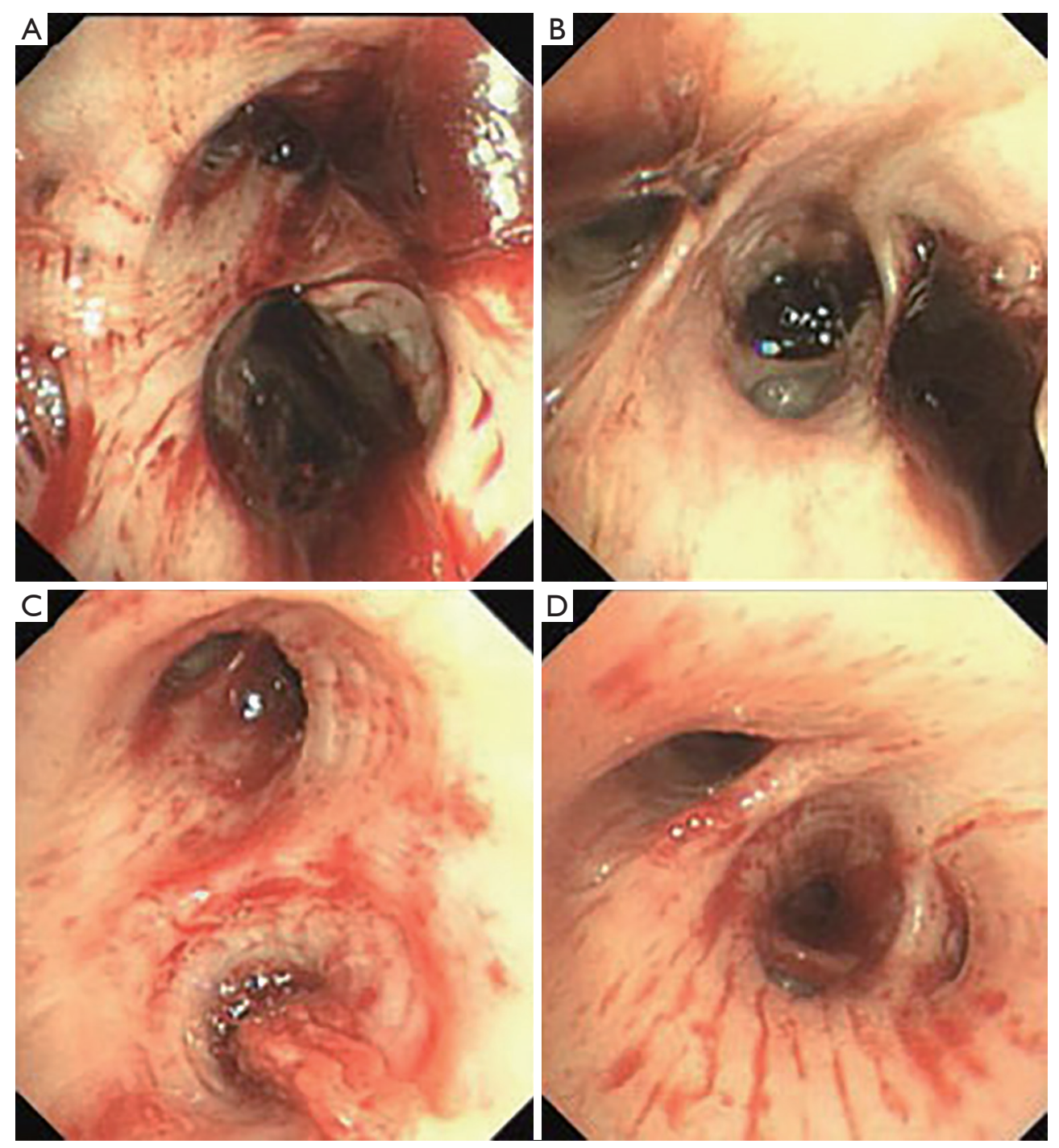

Figure 5 Rigid bronchoscopy images of the patient. Rigid bronchoscope view showing blood clots in the left lower lobe bronchus (A) and the bronchus intermedius. (B) View of the bronchus after clots were removed with cryoprobes and intermittent suctioning (C, D). 


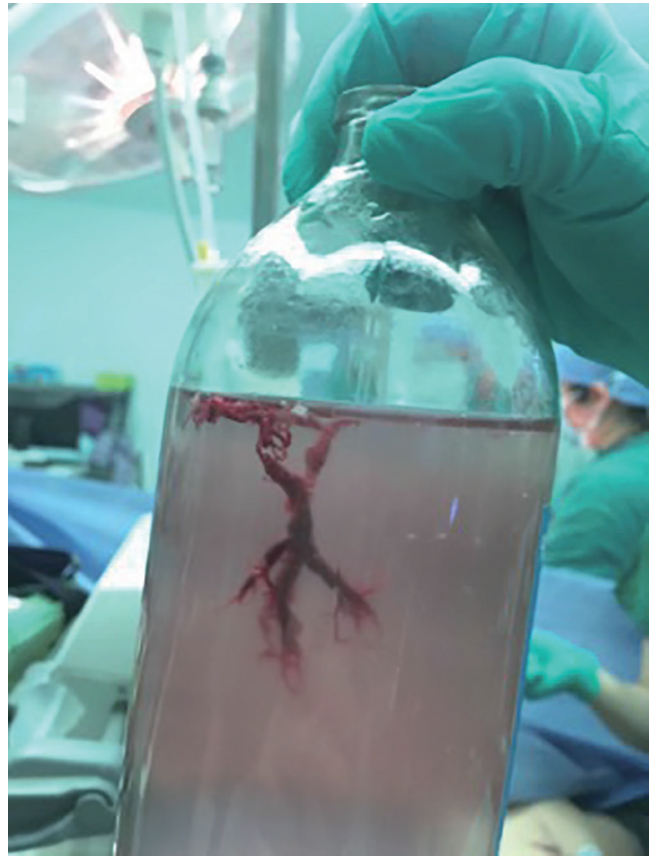

Figure 6 The blood clot extracted with cryoprobes reveal a bronchial cast.

tortuosity of the bilateral bronchial arteries. Distal to these, patchy staining and a disordered vascular mass were observed (Figure $7 A$ ). The endobronchial bleeding was localized, and the artery was embolized with gelatin sponge particles (350-560 $\mu \mathrm{m})$ (Figure 6B). After embolization, the bleeding stopped (Figure 7B), and endotracheal extubation was achieved on the second postpartum day after bedside bronchoscopy confirmed no active bleeding; the patient was then transferred from the ICU to the maternity ward on the third postpartum day. Additionally, the cardiologist recommended $25 \mathrm{mg}$ of oral sildenafil 3 times daily to decrease pulmonary vascular resistance. The patient was discharged home on the 10th postpartum day. Eleven months following the procedure, this patient had experienced no further episodes of hemoptysis.

The timelines for the 2 cases are provided in Figures 8 and 9.

\section{Ethical statement}

All procedures performed in studies involving human participants were in accordance with the ethical standards of the institutional and/or national research committee(s) and with the Helsinki Declaration (as revised in 2013). Written informed consent was obtained from both patients.
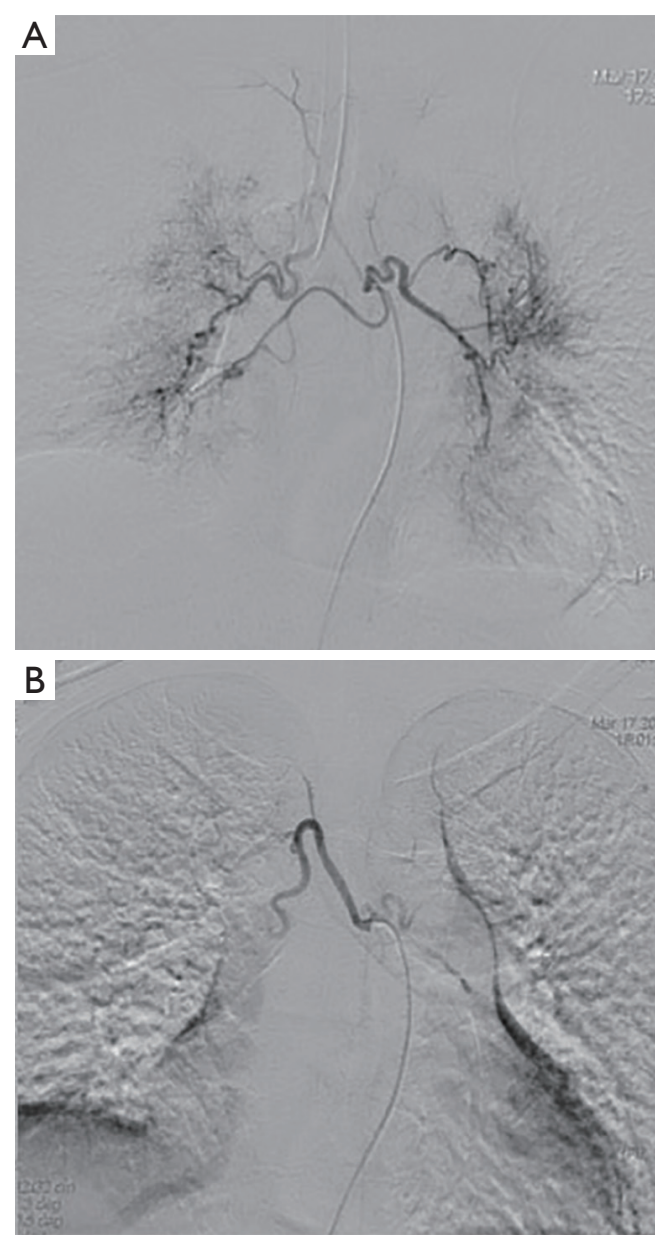

Figure 7 Selective angiograms of the bronchial artery before and after embolization. (A) Pre-embolization: Selective catheterization of the bronchial arteries shows enlargement and tortuosity of bilateral bronchial arteries; distal to this, patchy staining and disordered vascular mass were revealed. (B) Post-embolization: Selective catheterization of the bronchial arteries shows the absence of patchy staining.

\section{Discussion}

The manifestations of case 1 indicated a diagnosis of bronchial-to-pulmonary artery malformation (BPAM), which features multiple vascular communications between the bronchial and pulmonary circulations (10). Case 1 denied previous lung disease, although the white blood count was slightly elevated while the procalcitonin (PCT) was negative; thus, this case may fit the criteria for the congenital type of hemoptysis. It is well documented that maternal blood volume increases sharply during pregnancy due to increased cardiac output, sodium retention, and hormonally related 


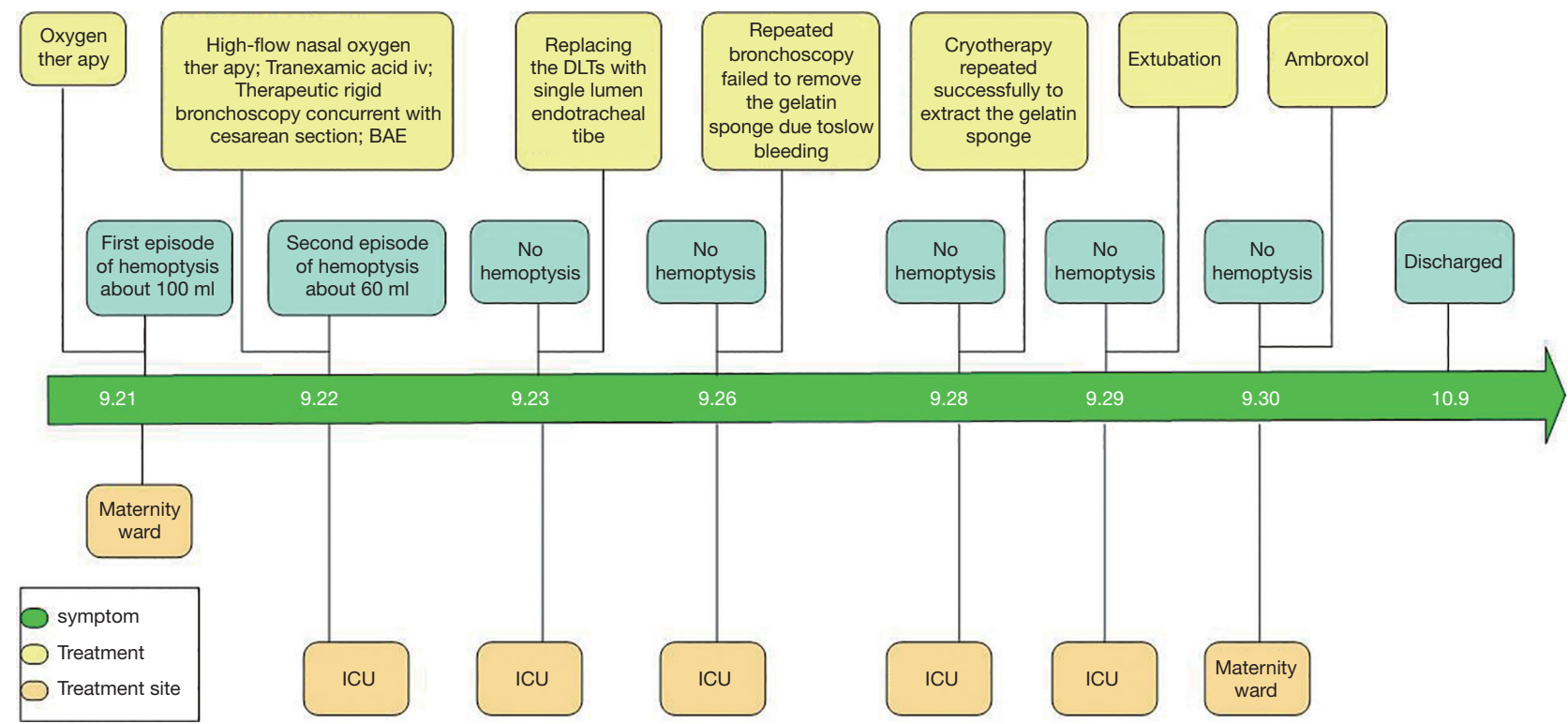

Figure 8 Timeline of case 1.

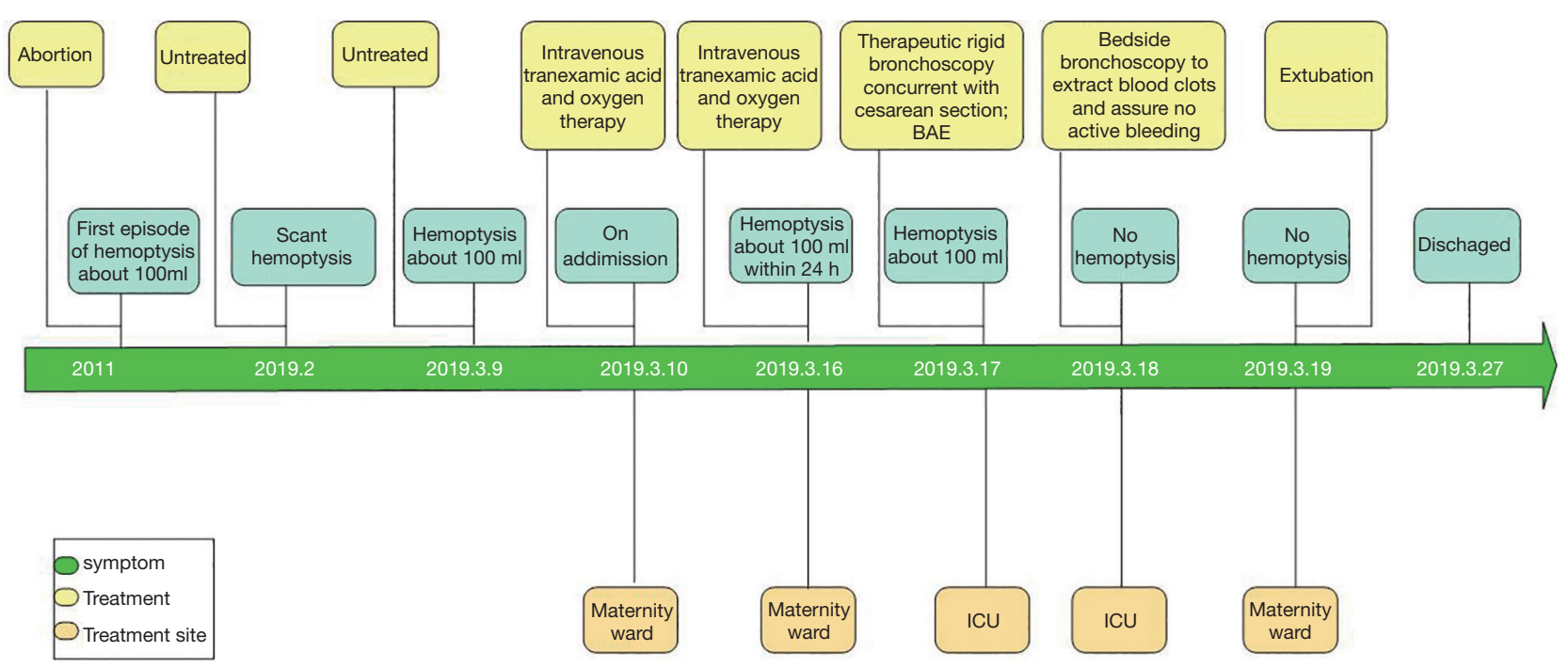

Figure 9 Timeline of case 2.

vascular change. More specifically, the plasma volume can increase to an average of $1,100-1,600 \mathrm{~mL}$, which is $30-50 \%$ above the normal level, plateauing for the last 8 weeks of pregnancy (11). Significantly increased blood flow during pregnancy can lead to vasodilation and collateral circulation, resulting in increased pressure in the aberrant focal lesion, which ruptures and bleeds, causing hemoptysis.

Hemoptysis is a severe and rare complication of pulmonary arterial hypertension (PAH) and is associated with a poor prognosis and a high mortality rate, even in hemodynamically stable patients (12). There was no indication for the diagnosis of chronic thromboembolic pulmonary hypertension (CTEPH) for our patient presented in case 2, as she did not have a history of venous thromboembolism and had a reasonable level of D-dimer. Meanwhile, the evidence for secondary etiologies of PAH including anti-nuclear antibody (ANA), endonuclear antibody (ENA) panel, antineutrophil cytoplasm antibody 
(ANCA), C3, C4, lupus anticoagulant, HIV, and hepatic virus antibodies were all negative. Furthermore, since the case 2 patient presented with evidence of PAVM and had a family history of hemoptysis, we considered a diagnosis of hereditary hemorrhagic telangiectasia accompanied by pulmonary arterial hypertension. Thus, a further contrastenhanced CT and a gene mutation test were recommended. However, the patient refused the tests for financial reasons. Notably, since physiological changes during pregnancy put considerable strain on the right ventricle and can lead to right ventricular failure, patients with $\mathrm{PAH}$ should be advised at the time of their diagnosis that pregnancy is not recommended due to the high maternal and fetal risks (13).

The initial approach in the management of massive hemoptysis is early control of the hemorrhage and simultaneous airway protection. Immediate lateralization performed with the bleeding side down is the first step to preventing the aspiration of blood to the contralateral lung (1). Reviewing the literature, we noticed that 21 cases of massive hemoptysis during pregnancy had been reported (Table S1). Their age ranged from 19 to 35 years with the second and third trimester predominating (90.4\%), which is consistent with the hemodynamic changes in pregnancy. The most common etiologies of these patients were infection (23.8\%), tumor (23.8\%), autoimmune disease (14.3\%), and idiopathic hemoptysis probably with a hormonal role (19.0\%). Flexible bronchoscopy was used in $15(71.4 \%)$ patients, and a combination of rigid and flexible bronchoscopy was used in 3 patients (20\%). Rigid bronchoscopy was used in 6 patients (28.6\%), and a subsequent BAE was used in two patients $(33.3 \%)$ prenatally. Three cases resulted in termination of the pregnancy. Pregnancy outcomes revealed two maternal deaths and three cases of fetal death. Of the patients with accessible follow-up information, spanning 1-96 months, none reported further hemoptysis, and all of the infants were reportedly well.

Based on the literature review, we could not find any case managed by concurrent cesarean section and therapeutic rigid bronchoscopy followed by BAE after surgery. These are the highlights of our cases. For our patients, medications such as phentolamine are not recommended for low blood pressure, and pituitrin is forbidden for use in contracting the uterine smooth muscle. Although BAE was recommended as the first-line therapy based on its effectiveness in both finding the source of bleeding and controlling hemoptysis (14), the contrast media can cross the human placenta and harm the fetus; thus, cesarean section under general anesthesia was performed first, followed by planned BAE. Early visualization with a flexible bronchoscope can be helpful in selective intubation, bronchial blocker placement, or for therapeutic purposes. However, the size of the suction port with a flexible bronchoscopy is inadequate. Rigid bronchoscopy is a more effective tool for the isolation of the mainstem bronchus while ensuring ventilation, for localization of the source of bleeding, and for maintaining an open airway to assist in the application of the interventional bronchoscopy methods (15) in order to prevent potential massive hemoptysis during the cesarean section. For these reasons, we chose to perform therapeutic rigid bronchoscopy simultaneously with the cesarean section. BAE is also recommended to urgently treat patients with massive cryptogenic hemorrhage that does not respond to endobronchial management. To minimize the adverse effects of the contrast media on the fetus, we performed BAE after the cesarean section. Furthermore, to prevent another episode of hemoptysis, our patients were intubated with a DLT before the embolization, allowing for the isolation and ventilation of the normal lung and preventing aspiration from the potential bleeding side. After 24-hour observation to ensure there was no persistent bleeding, the DLTs were replaced with a single-lumen endotracheal for ventilation.

There are certain risks of performing bronchoscopy during massive hemoptysis that are related to sedation, including hypoventilation, airway vulnerability, and aspiration (16). However, bronchoscopic management is required to localize the site of bleeding, guide angiography, and perform embolization and bronchoscopic interventions (17). As the fetus is sensitive to maternal hypoxia and hypotension, close monitoring of pregnant patients is necessary. This involves an initial assessment of the patient's medical history, continuous monitoring of intermittent sphygmomanometry, cardiac rhythm and rate, and pulse oximetry (18).

The detailed descriptions of these 2 cases are the strengths of this report, but an obvious limitation is the lack of specific RHC information in case 2. Since there is no evidence of secondary $\mathrm{PH}$, we concluded that our case 2 patient can be classified as group $1 \mathrm{PH}$.

The highlights of our cases are the successful control of hemorrhage and airway management of massive hemoptysis through rigid bronchoscopy with HFJV under intravenous general anesthesia during cesarean section, as well as the combination of BAE, the use of DLTs prior to ETTs, and flexible bronchoscopy and HFNC in the perioperative period. Patients should be monitored in the ICU to provide them with comprehensive and effective life support, 
while active allowing cooperation with other specialists to jointly treat critically ill patients should be pursued. Crossspecialty and multidisciplinary efforts are also the keys to the successful management of massive hemoptysis.

\section{Acknowledgments}

We thank the patients for permitting us to share their cases. Funding: None.

\section{Footnote}

Reporting Checklist: The authors have completed the CARE reporting checklist. Available at http://dx.doi.org/10.21037/ atm-20-2502

Conflicts of Interest: All authors have completed the ICMJE uniform disclosure form (available at http://dx.doi. org/10.21037/atm-20-2502). The authors have no conflicts of interest to declare.

Ethical Statement: The authors are accountable for all aspects of the work in ensuring that questions related to the accuracy or integrity of any part of the work are appropriately investigated and resolved. All procedures performed in studies involving human participants were in accordance with the ethical standards of the institutional and/or national research committee(s) and with the Helsinki Declaration (as revised in 2013). Written informed consent was obtained from the patient.

Open Access Statement: This is an Open Access article distributed in accordance with the Creative Commons Attribution-NonCommercial-NoDerivs 4.0 International License (CC BY-NC-ND 4.0), which permits the noncommercial replication and distribution of the article with the strict proviso that no changes or edits are made and the original work is properly cited (including links to both the formal publication through the relevant DOI and the license). See: https://creativecommons.org/licenses/by-nc-nd/4.0/.

\section{References}

1. Davidson K, Shojaee S. Managing Massive Hemoptysis. Chest 2020;157:77-88.

2. Radchenko C, Alraiyes AH, Shojaee S. A systematic approach to the management of massive hemoptysis. J Thorac Dis 2017;9:S1069-86.
3. Jean-Baptiste E. Clinical assessment and management of massive hemoptysis. Crit Care Med 2001;29:1098.

4. Rocha MP, Guntupalli KK, Moise KJ Jr. Massive hemoptysis in Takayasu's arteritis during pregnancy. Chest 1994;106:1619-22.

5. Flight WG, Tang I, Gates A, et al. Massive hamoptysis and ventilatory failure in pregnancy. Thorax 2019;74:818-20.

6. Chhajed PN, Kate A, Chaudhari P, et al. Massive hemoptysis during pregnancy. J Assoc Physicians India 2011;59:660-2.

7. Masukume G, Sengurayi E, Moyo P, et al. Massive hemoptysis and complete unilateral lung collapse in pregnancy due to pulmonary tuberculosis with good maternal and fetal outcome: a case report. BMC Res Notes 2013;6:335.

8. Downs TW, Chao CR. Massive hemoptysis in pregnancy treated with bronchial artery embolization. Am J Perinatol 1997;14:51-3.

9. Riley DS, Barbe`r MS, Kienle GS, et al. CARE 2013 Explanations and Elaborations: Reporting Guidelines for Case Reports. J Clin Epidemiol 2017;89:218-35.

10. Uchiyama D, Fujimoto K, Uchida M, et al. Bronchial arteriovenous malformation: MDCT angiography findings. AJR Am J Roentgenol 2007;188:W409-11.

11. Metcalfe J, Ueland K. Maternal cardiovascular adjustments to pregnancy. Prog Cardiovasc Dis 1974;16:363-74.

12. Żyłkowska J, Kurzyna M, Pietura R, et al. Recurrent hemoptysis: an emerging life-threatening complication in idiopathic pulmonary arterial hypertension. Chest 2011;139:690-3.

13. Olsson KM, Channick R. Pregnancy in pulmonary arterial hypertension. Eur Respir Rev 2016;25:431-7.

14. Panda A, Bhalla AS, Goyal A. Bronchial artery embolization in hemoptysis: a systematic review. Diagn Interv Radiol 2017;23:307-17.

15. Conlan AA, Hurwitz SS. Management of massive haemoptysis with the rigid bronchoscope and cold saline lavage. Thorax 1980;35:901-4.

16. Bahhady IJ, Ernst A. Risks of and recommendations for flexible bronchoscopy in pregnancy: a review. Chest 2004;126:1974-81.

17. Sakr L, Dutau H. Massive hemoptysis: an update on 
the role of bronchoscopy in diagnosis and management. Respiration 2010;80:38-58.

18. Cappell MS. The fetal safety and clinical efficacy

Cite this article as: Yang $\mathrm{X}, \mathrm{Ma} \mathrm{W}$, Shi $\mathrm{X}$, Sun $\mathrm{X}$, Wei Y, Yan Z, Li S. Novel approach of rigid bronchoscopy concurrent with cesarean section and bronchial arterial embolism for patients with massive hemoptysis during pregnancy: case reports and literature review. Ann Transl Med 2021;9(7):582. doi: $10.21037 /$ atm-20-2502 of gastrointestinal endoscopy during pregnancy.

Gastroenterol Clin North Am

2003;32:123-79. 
Supplementary

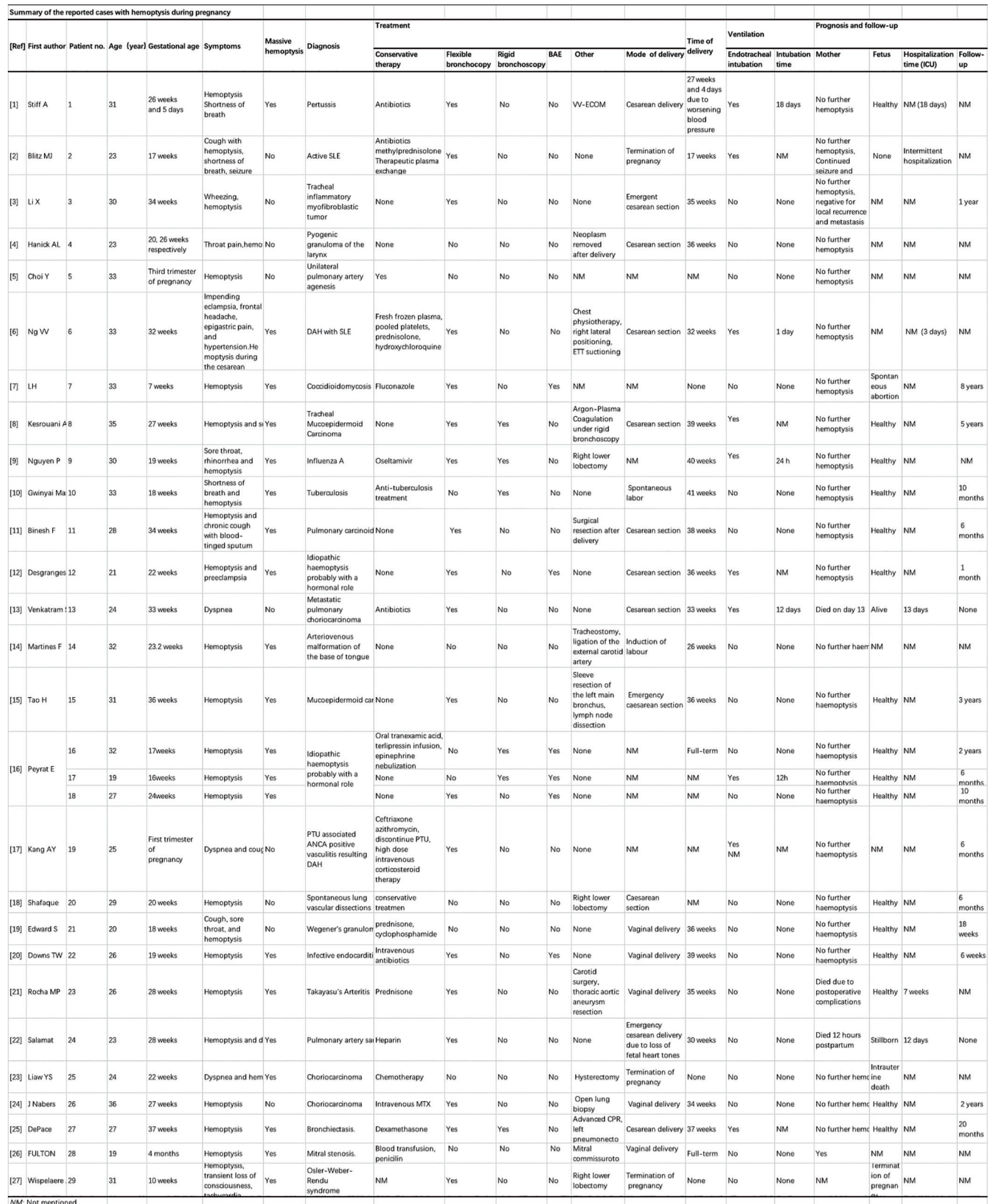

(c) Annals of Translational Medicine. All rights reserved. 


\section{References}

19. Stiff A, Harrison R, Palatnik A. Case report of massive hemoptysis in pregnancy requiring veno-venous extracorporeal membrane oxygenation. J Obstet Gynaecol Res. 2019

20. Blitz MJ, Fleischer A. Severe Maternal Morbidity Associated with Systemic Lupus Erythematosus Flare in the Second Trimester of Pregnancy. Case Rep Obstet Gynecol. 2018 May

21. Ng VV, Shah MK, Oh TT, et al. Massive Hemoptysis Through Endotracheal Tube During Emergency: A Case Report and Literature Review. A A Case Rep. 2017 Sep 1

22. Labuschagne H, Burns C, Martinez S. Coccidioidomycosis in pregnancy: Case report and literature review of associated placental lesions. Case Rep Womens Health. 2016 Oct 20

23. Kesrouani A, Dabar G, Rahal S, et al. Treatment of tracheal mucoepidermoid carcinoma by argon plasma coagulation. Int Surg. 2015 May

24. Nguyen P, Weber F, Mahone M. A 30-year-old pregnant patient with massive haemoptysis and influenza A: Atypical presentation of a common pathogen. Obstet Med. 2013 Dec

25. Gwinyai Masukume, Elton Sengurayi, Phinot Moyo. Massive hemoptysis and complete unilateral lung collapse in pregnancy due to pulmonary tuberculosis with good maternal and fetal outcome: a case report.

26. Binesh F, Samet M, Bovanlu TR. A case of pulmonary carcinoid tumour in a pregnant woman successfully treated with bronchoscopic (electrocautery) therapy. BMJ Case Rep. 2013 Apr 22

27. Desgranges FP, Berthelot AL, Gamondes D, et al. Massive recurrent haemoptysis in a pregnant woman with preeclampsia.

28. Martines F, Immordino V. Arteriovenous malformation of the base of tongue in pregnancy: case report. Acta Otorhinolaryngol Ital. 2009 Oct

29. Tao H, Sano Y, Yamane M, et al. Bronchial mucoepidermoid carcinoma with recurrent hemoptysis in a pregnant. Surg Today. 2008

30. Peyrat E, Chabbert V, Escamilla R. Idiopathic hemoptysis in pregnant women: A distinct entity? Respir Med. 2007 Oct

31. Downs TW, Chao CR. Massive hemoptysis in pregnancy treated with bronchial artery embolization. Am J Perinatol. 1997 Jan

32. Rocha MP, Guntupalli KK, Moise KJ Jr, et al. Massive Hemoptysis in Takayasu's Arteritis During Pregnancy. Chest. 1994 Nov.

33. Salamat SM, Landy HJ, O'Sullivan MJ. Pulmonary artery sarcoma in a pregnant woman: report of a case. Obstet Gynecol. 1994 Oct

34. Liaw YS, Yang PC, Yuan A. Ultrasonography and color Doppler imaging of metastatic pulmonary choriocarcinoma. Chest. 1993 Nov;104(5):1600-1.

35. DePace NL, Betesh JS, Kotler MN. Postmortem' cesarean section with recovery of both mother and offspring. JAMA. 1982 Aug 27

36. Fulton WF, Smith G. Severe hemoptysis during pregnancy treated by mitral commissurotomy. Circulation.1958 Feb;17(2):204-7.

37. Wispelaere JF, Trigaux JP, Weynants P. Systemic supply to a pulmonary arteriovenous malformation: potential explanation for recurrence. Cardiovasc Intervent Radiol. 1996 Jul-Aug;19(4):285-7. 